\title{
Experimental Characterization of a Load Modulated Balanced Amplifier with Simplified Input Power Splitter
}

\author{
David Collins*, Roberto Quaglia*, Jeff Powell ${ }^{\dagger}$, Steve Cripps* \\ * Cardiff University, Queen's Buildings, Cardiff, UK \\ collinsdj1@ cardiff.ac.uk \\ † Skyarna Ltd, Halesowan, West Midlands, U.K
}

\begin{abstract}
This paper presents the experimental characterization of a load modulated balanced amplifier for base station applications using a single input configuration. An off-the-shelf power splitter is used with coaxial cables of different length to divide the input power between the branches. The effect of the phase offset is assessed experimentally by $\mathrm{CW}$ single tone and modulated signal measurements, and the results are discussed. With a proper selection of cable length, the amplifier achieves a CW output power of $48 \mathrm{dBm}$ and a $6 \mathrm{~dB}$ back-off efficiency of $44 \%$ at $1.9 \mathrm{GHz}$, while it maintains an output power higher than $47.5 \mathrm{dBm}$ and a back-off efficiency higher than $32 \%$ between 1.8 and $2.3 \mathrm{GHz}$. The amplifier, tested with a Long Term Evolution signal, can be linearized with a digital predistorter to comply with spectral regrowth requirements.
\end{abstract}

Index Terms-Balanced power amplifiers, load modulation, gallium nitride

\section{INTRODUCTION}

The need for transmitting more and more information wirelessly has required the optimization of the frequency spectrum resources, leading to the use of high modulation orders together with very narrow band-guards between different frequency channels. Both these conditions pose stringent linearity requirements to the transmitters in order not to deteriorate the in-band distortion - usually quantified in terms of Error Vector Magnitude (EVM) - and avoid excessive spectral regrowth quantified by the Adjacent Channel Leakage Ratio (ACLR). To meet those requirements, it is often necessary to trade-off the power amplifier (PA) efficiency by operating it in back-off to avoid clipping and saturation. A widely adopted solution for improving the linearity vs. efficiency trade-off is the Doherty PA, introduced back in 1936[1] for AM transmissions, and revived for modern communications [2], [3]. The Doherty is based on load-modulation, meaning that the load seen by the active device changes instantaneously with the modulated signal, helping to maintain an optimal voltage swing and consequently a good efficiency also for reduced input power.

To overcome one of the main issues of the Doherty PA, i.e., its limited bandwidth, a recently introduced novel PA configuration, the Load Modulated Balanced Amplifier (LMBA) [4], has been proposed as a possible PA for modulated signal amplification [5]. The results shown by the LMBA are very promising and compare well with the state of the art in terms of bandwidth, output power and also linearity. However, the LMBA is in general based on a dual-input configuration that might be considered, in some cases, as a complication to the transmitter that cannot be afforded, despite the fact that dualinput PAs, such as dual-input Doherty PAs, have demonstrated great potential [6], [7]. Single-input LMBAs have been already demonstrated [8], [9], and a comparative analysis between a dual-input and an emulated single input configuration has been presented in [10].

In this paper, for the first time, an LMBA designed for dual input operation has been characterised in a single input configuration by using a simple off-the shelf input splitter. The impact of change in relative delay between the inputs is analysed experimentally, and the results are critically commented.

\section{LOAd Modulated Power AMPlifier (LMBA)}

The LMBA is based on a balanced pair of amplifiers (BPAs) with an RF control signal power (CSP) injected at the output isolated port permitting controlled load modulation. Over the bandwidth of the 3-dB couplers of the BPA, the CSP signal can modulate the load in any direction from the coupler home impedance, and the CSP power always adds up at the output, with a clear advantage with respect to a Doherty PA [5]. In

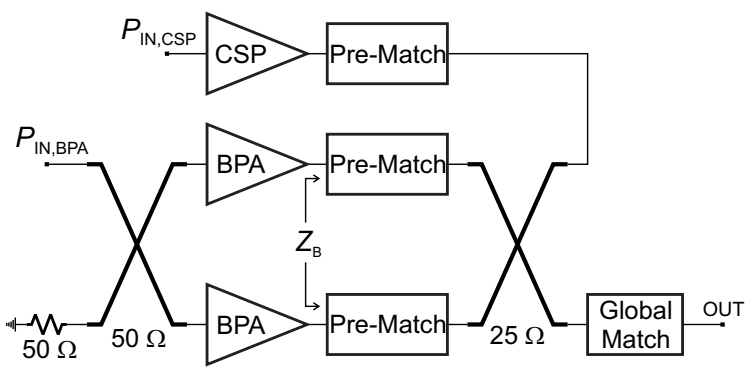

Fig. 1. Block diagram of the LMBA characterised in this paper.

the LMBA characterised in this paper (schematic diagram in Fig. 1), the BPA transistors load $Z_{\mathrm{B}}$ was prematched to a high efficiency optimum $Z_{\mathrm{opt}, \mathrm{OBO}}$ at around half of the maximum power deliverable by the device, so that when the CSP was inactive the amplifiers achieved a first efficiency peak at backoff power. The CSP injected power, in this case amplified by a CSP buffer stage, moved the load to the optimum for maximum power $Z_{\mathrm{opt}, \mathrm{SAT}}$ and also contributed to the output power. In particular, the ratio $\rho_{\mathrm{C}}=P_{\mathrm{IN}, \mathrm{CSP}} / P_{\mathrm{IN}, \mathrm{BPA}}$ and the relative phase $\phi_{\mathrm{C}}$ between inputs must be properly set, as a function of $P_{\mathrm{IN}, \mathrm{BPA}}$ to achieve the correct load modulation. 
If compared to a Doherty PA, the BPA acted as the main amplifier, and was biased in class $\mathrm{AB}$, while the CSP worked as auxiliary and biased in class $\mathrm{C}$. The impedance of the output coupler was chosen as $25 \Omega$ to reduce the impedance ratio for the prematching. For this reason, global matching was needed to feed the $50 \Omega$ output port. The devices are packaged $25 \mathrm{~W}$ GaN HEMTs from Wolfspeed, and the designed LMBA targeted the $1.7-2.5 \mathrm{GHz}$ band.

Fig. 2 (left) shows the simulated load modulation at $2.2 \mathrm{GHz}$ as a function of $\rho_{\mathrm{C}}$ and $\phi_{\mathrm{C}}$. For low CSP injection, the prematching sets the load close to $Z_{\mathrm{opt}, \mathrm{OBO}}$, while for increasing CSP power a correct phase adjustment is necessary to bring the load to the optimum $Z_{\mathrm{opt}, \mathrm{SAT}}$.
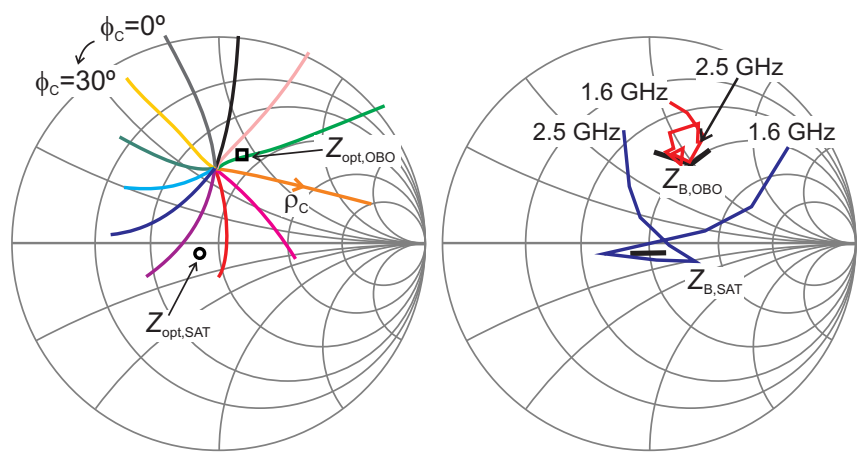

Fig. 2. Left: Dual-input LMBA; simulated $Z_{\mathrm{B}}$ at $2.3 \mathrm{GHz}$ as function of $\rho_{\mathrm{C}}$ and $\phi_{\mathrm{C}}$. Right: Single-input LMBA; simulated $Z_{\mathrm{B}}$ at back-off (red) and saturation (blue) vs. frequency; black traces show the position of the corresponding target optimum loads. Smith Charts normalised at $12 \Omega$.

\section{SingLE InPUT LMBA}

The load modulation effectively improves the LMBA efficiency if its amount and direction are properly controlled as a function of the instantaneous input power. With separate $\mathrm{RF}$ inputs, this can be achieved by controlling independently the modulated signals. The LMBA used in this paper was characterized with separate inputs in [5], achieving on the $1.7-2.5 \mathrm{GHz}$ bandwidth an output power higher than $48 \mathrm{dBm}$ and a back-off efficiency higher than $43 \%$. This required both amplitude and phase independent controls, and such freedom was also beneficial with modulated signals, where a quadratic function was applied to determine the CSP input. However, where the complexity of such a solution is not acceptable, it is possible to derive the CSP input by splitting or probing the BPA input. In [8], the input has been divided by a $3 \mathrm{~dB}$ coupler and the phase has been adjusted to address the desired behaviour at single frequency, i.e., $800 \mathrm{MHz}$. A similar solution has been then proposed in [9] where, however, thanks to broadband matching and a detailed design of a filter-based delay network, an octave bandwidth has been achieved.

In this paper, the strategy has been to try to characterise the LMBA designed for independent inputs with an external splitter based on off-the shelf power divider and coaxial cables of different lengths to adjust $\phi_{\mathrm{C}}$, see Fig. 3. A preliminary simulation has been used to determine an initial value for $\phi_{\mathrm{C}}$.

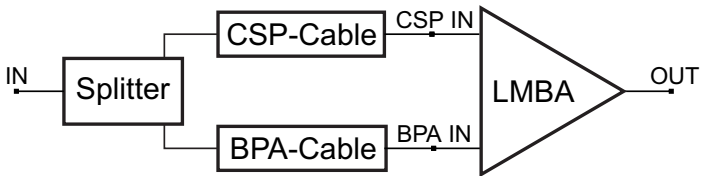

Fig. 3. Single input LMBA approach.

By observing the optimum phase setting for the dual-input simulations, a reasonable approximation was given by using a transmission line delay of $162 \mathrm{ps}$ on the BPA side. By simulating the LMBA with this setting, the bandwidth resulted limited in the $1.8-2.3 \mathrm{GHz}$ with a reduced performance compared to the dual-input, but still reasonable. Fig. 2 (right) shows the simulated $Z_{\mathrm{B}}$ at back-off and saturation vs. frequency in this single input configuration. The saturated load remains close to the corresponding optimum in the frequency range 1.8$2.3 \mathrm{GHz}$, while the deviation is quite large outside this band.

\section{EXPERIMENTAL CHARACTERIZATION}

The single-input LMBA has been characterised in $\mathrm{CW}$ single tone and modulated signal conditions. The measurement setup (picture in Fig. 4) is based on a vector signal generator feeding, through a driver amplifier, the LMBA. The input and output power was measured with power meters. A heterodyne receiver, based on a passive mixer and a digital oscilloscope, was used to detect the modulated signal in the system level characterization.

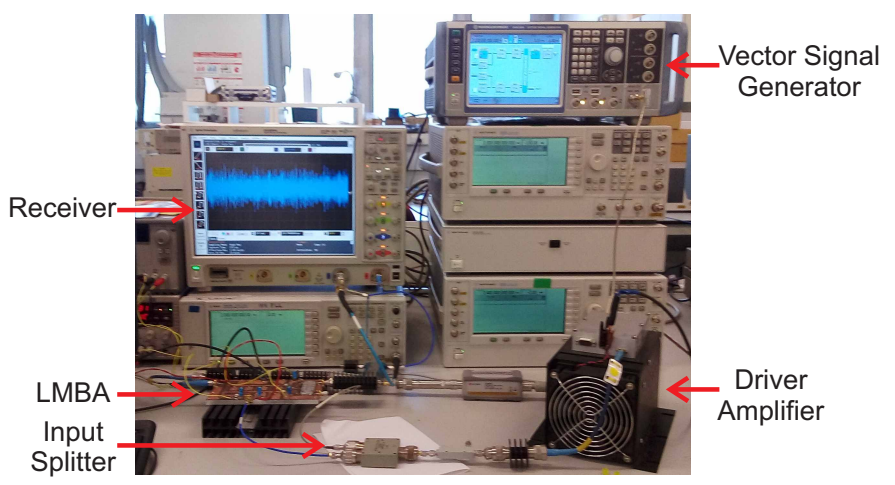

Fig. 4. Picture of the measurement system.

$\mathrm{CW}$ single tone measurements were performed in the 1.6$2.4 \mathrm{GHz}$ band with $100 \mathrm{MHz}$ steps. Two coaxial cables whose length difference equals a delay difference of $162 \mathrm{ps}$ (referred to as $\left.\phi_{\mathrm{C} 0}\right)$ were used to connect the BPA and CSP inputs to the input power divider (ZAPD-4 from MiniCircuits). Then, a SMA adapter with a delay around $64 \mathrm{ps}$ was used to increase the delay on the CSP side (measurement condition referred to as $\phi_{\mathrm{C}+}$ ) and the BPA side (referred to as $\phi_{\mathrm{C}_{-}}$) to evaluate the effect on performance and experimentally verify if the optimum setting determined in simulations was also the best in measurements. The BPAs were biased at drain voltage of $28 \mathrm{~V}$ and total drain current of $60 \mathrm{~mA}$, while the CSP was biased at 


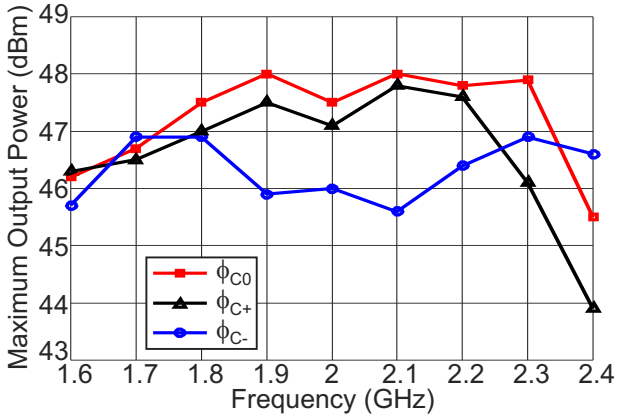

Fig. 5. Maximum output power vs. CW frequency for different delays.

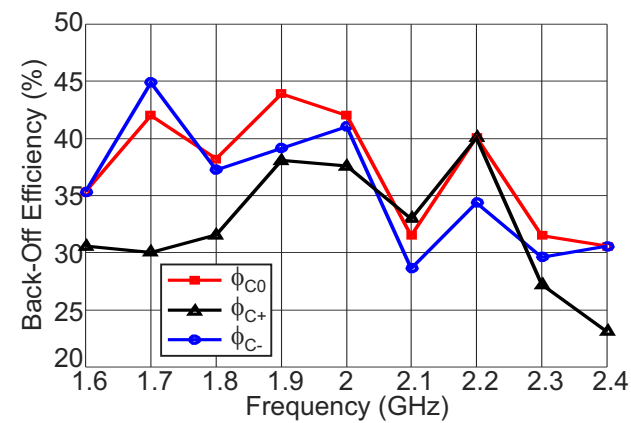

Fig. 6. Back-off efficiency vs. CW frequency for different delays.

$28 \mathrm{~V}$ drain and $-7 \mathrm{~V}$ gate. Fig. 5 and Fig. 6 shows the maximum output power and the $6 \mathrm{~dB}$ back-off efficiency, respectively, vs. frequency and for different delay settings. The $\phi_{\mathrm{C} 0}$ settings allowed the best performance over the bandwidth, suggesting that simulations were fairly reliable in indicating the correct phase settings. Fig 7 shows the output power, gain, maximum and back-off efficiency vs. frequency with the $\phi_{\mathrm{C} 0}$ setting. At $1.9 \mathrm{GHz}$, the LMBA showed its best performance with maximum power of $48 \mathrm{dBm}$, back-off efficiency of $44 \%$, and gain of $7 \mathrm{~dB}$. Over the $1.8-2.3 \mathrm{GHz}$ band, the output power was larger than $47.5 \mathrm{dBm}$ and the back-off efficiency was higher than $32 \%$. These results are reduced if compared to the dualinput results presented in [5], but still compare reasonably well

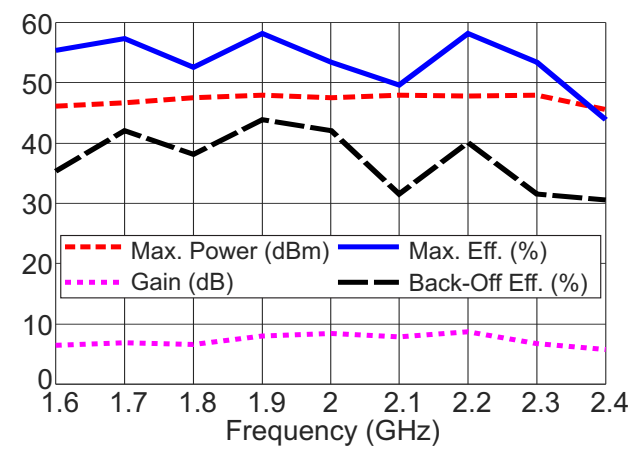

Fig. 7. Output power, gain, maximum efficiency and back-off efficiency vs. $\mathrm{CW}$ frequency for the single input LMBA. with the literature.
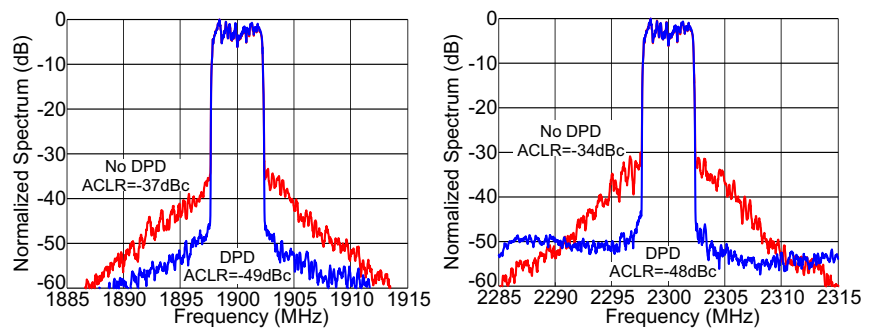

Fig. 8. Measured spectrum for the single input LMBA with $5 \mathrm{MHz}$ LTE test signal at average output power of $39 \mathrm{dBm}$, without and with predistortion. Left: $1900 \mathrm{MHz}$, average efficiency of $42 \%$. Right: $2300 \mathrm{MHz}$, average efficiency of $34 \%$.

Finally, to test the linearizeability of the LMBA, that could be more critical in a single-input configuration [10], an LTE $5 \mathrm{MHz}$ channel signal with PAPR of around $9 \mathrm{~dB}$ is used. Due to the relaxed thermal stress, the LMBA showed 1-1.5 dB higher maximum power across the bandwidth compared to single-tone measurements. By applying a memory polynomial digital predistortion, the ACLR could be brought well below the $-45 \mathrm{dBc}$ standard requirement, as shown in Fig. 8 .

\section{CONCLUSION}

A load modulated balanced amplifier, originally designed for dual-input operation, has been characterized using a simple single-input configuration. Despite the expected reduction in bandwidth, the amplifier still shows good results both in CW single tone and modulated signal conditions, demonstrating the versatility of the hardware.

\section{REFERENCES}

[1] W. Doherty, "A new high efficiency power amplifier for modulated waves," Proc. IRE, vol. 24, no. 9, pp. 1163 - 1182, Sep. 1936.

[2] A. Grebennikov and S. Bulja, "High-Efficiency Doherty Power Amplifiers: Historical Aspect and Modern Trends," Proc. IEEE, vol. 100, no. 12, pp. 3190 -3219, Dec. 2012.

[3] V. Camarchia, et al., "The Doherty power amplifier: Review of recent solutions and trends," IEEE Trans. Microw. Theory Techn., vol. 63, no. 2, pp. 559-571, Feb. 2015.

[4] D. J. Shepphard, et al., "An efficient broadband reconfigurable power amplifier using active load modulation," IEEE Microw. Wireless Compon. Lett., vol. 26, no. 6, pp. 443-445, June 2016.

[5] R. Quaglia and S. Cripps, "A load modulated balanced amplifier for telecom applications," IEEE Trans. Microw. Theory Techn., vol. 66, no. 3, pp. 1328-1338, Mar. 2018.

[6] K. Bathich, et al., "Frequency response analysis and bandwidth extension of the Doherty amplifier," IEEE Trans. Microw. Theory Techn., vol. 59, no. 4, pp. $934-944$, Apr. 2011.

[7] C. M. Andersson, et al., "A 1-3-GHz digitally controlled dual-RF input power-amplifier design based on a Doherty-outphasing continuum analysis," IEEE Trans. Microw. Theory Techn., vol. 61, no. 10, pp. $3743-$ 3752, Oct. 2013.

[8] P. Pednekar and T. Barton, "RF-input load modulated balanced amplifier," in Microwave Symposium (IMS), 2017 IEEE MTT-S International, Jun. 2017, pp. 1-3.

[9] P. H. Pednekar, et al., "RF-Input load modulated balanced amplifier with octave bandwidth," IEEE Trans. Microw. Theory Techn., vol. 65, no. 12, pp. 5181-5191, 2017.

[10] R. Quaglia, et al., "Analysis and characterization of a load modulated balanced amplifier for base-station applications," in 2018 11th German Microwave Conference (GeMiC), March 2018, pp. 1-4. 